\title{
Integration of a Hewlett-Packard minicomputer with digital-logic controlled behavioral laboratories*
}

\author{
DAVID K. BILLINGS and DOUGLAS P. FERRARO \\ University of New Mexico, Albuquerque, New Mexico 87137
}

\begin{abstract}
A Hewlett-Packard on-line minicomputer controller for a new primate laboratory was integrated with existing equipment in digital-logic controlled rat and pigeon laboratories so as to maximize the utility of the separate systems.
\end{abstract}

Instrumenting a new nonhuman primate laboratory, in conjunction with a need to strengthen the research capabilities of existing digital-logic controlled pigeon and rat laboratories, prompted the consideration of a minicomputer as an alternative to digital logic. Programming of the primate experiments could have been accomplished by the acquisition of additional digital logic. Such an increase would have strengthened the other laboratories but would not have appreciably increased the flexibility of these systems. Furthermore, this approach would have only compounded the immediate problems of data collection and reduction.

The general advantages of a minicomputer have been discussed previously (e.g., Sidowski, 1970, 1972), and several Es have reported success specifically in controlling primate behavioral laboratories with on-line computers (Barensten \& Lockard, 1973; Davenport et al, 1972; Moise \& Jarrard, 1969). Nevertheless, the alternative of a computer was only acceptable if it could alleviate data reduction problems, perform on-line data collection functions for the pigeon and rat laboratories, and provide the needed instrumentation for the primate laboratory. Strict replacement of the existing digital logic with a sufficiently sized computer system was not economically feasible and would have resulted in some of the inappropriate uses of on-line computers noted by Uttal (1972).

The solution was to retain the digital logic and to integrate a Hewlett-Packard 2100A minicomputer with this equipment. This integration was accomplished by channeling to the digital logic those functions that could be better handled by the digital logic and leaving the computer free to perform only those functions most appropriately handled by it. Three general modes of computer use were established by routing input and output logic channels through a plug board. These modes were: complete on-line control of program contingencies, data acquisition, and immediate data

\footnotetext{
*This work was supported by NIMH Grant DA 00355. The authors thank John Gluck and Gerald Bemis for their assistance in the design and fabrication of the monkey chambers.
}

analysis; real time data acquisition and immediate data analysis with appropriate contingency control functions delegated to digital logic; and analysis of data stored on punched paper tape with contingency control and data acquisition responsibilities assigned completely to the digital logic.

\section{EQUIPMENT}

The primate equipment consists of four locally fabricated, sound attenuating, primate operant chambers. Each chamber contains five translucent plastic response keys arranged in the shape of a Greek cross. Mounted behind each key is an IEE in-line stimulus projector, which is used to project any of 12 different form or color stimuli onto the key. In addition, each chamber contains a retractable lever, a houselight, and a pellet dispenser.

Both the pigeon and the rat laboratories have an adequate amount of digital logic for most programming purposes, electromechanical impulse counters, a paper-tape reader, and a paper-tape punch. The operant chambers are three Lehigh Valley pigeon units and four Scientific Prototype rat chambers.

The computer hardware includes a Hewlett-Packard $2100 \mathrm{~A}$ minicomputer with $8 \mathrm{~K}$ memory (16-bit word, 1,024-word page), a programmable time base generator, an ASR 33 Teletype, a paper-tape photoreader (500 cps), and a paper-tape punch $(75 \mathrm{cps})$. Intercommunication with the operant chambers and digital logic is accomplished via two 16-bit duplex registers that are compatible with the logic levels of BRS Digibit units.

\section{INTERFACE}

Interfacing the computer to standard peripheral devices is accomplished by plug-in interface cards. The order in which the interface cards are plugged into the computer determines the priority of the peripheral device with respect to a multilevel priority interrupt structure. The interrupting capabilities of individual devices can be enabled or disabled by program instruction, as can the entire interrupt structure. The two Hewlett-Packard duplex registers that are used to interface the computer to the operant chambers and digital logic are at the end of the priority line.

Each duplex register provides a 16-bit buffer for input, a 16-bit buffer for output, a control bit, and a flag bit. The necessary voltage levels for the input bits are $-8 \mathrm{~V}$ for a $\operatorname{logic}$ one and $0 \mathrm{~V}$ for a logic zero. Output 


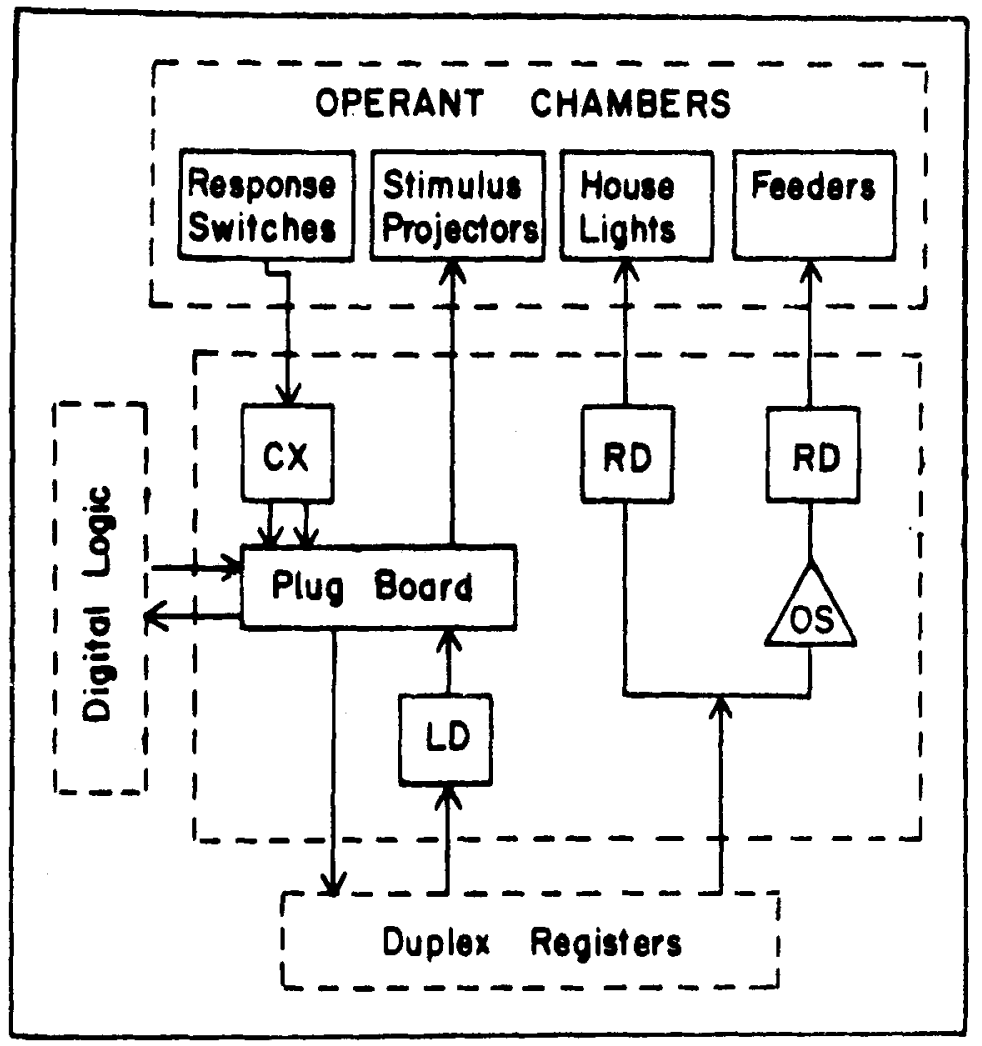

Fig. 1. Computer interface to operant chambers and digital logic.

voltage levels are $-12 \mathrm{~V}$ for a logic one and $0 \mathrm{~V}$ for a logic zero. The command signal is a level change from $-12 \mathrm{~V}$ to $0 \mathrm{~V}$, and the required flag signal is a change from $-8 \mathrm{~V}$ to $0 \mathrm{~V}$.

The state of both the control bit and the flag bit are under program control, while only the flag bit can be affected by an external source. Given that program instructions have enabled an interrupt by the duplex register, the setting of both the control bit and the flag bit brings about an interrupt to the memory location assigned to the duplex register. By changing the position of jumper wires, input bits can either follow the level changes of an external source or "latch in" data as the result of a command from the computer and the setting of the flag. Thus, data input can be accomplished either by a priority interrupt, a command and wait for completion procedure, or by making periodic status checks.

Switch closures from the primate response keys and retractable levers are converted to logic level signals by BRS input modifiers (CX-207). The digital logic unit will produce either a logic pulse of a specified duration or a continuous logic level which indicates the status of the switch. These logic outputs are channeled to a plug board where they may be interconnected to any of the 32 input channels of the two 16-bit duplex registers. The output side of these two registers have been hard wired to BRS digital logic units for control of events in the primate chambers. Four bits are wired to relay drivers (RD-206) for control of the houselights. A second 4 bits are wired to one-shots (OS-203) that provide a timed pulse to those relay drivers which operate the primate pellet dispensers. The remaining 24 bits are taken to the plug board through lamp drivers (LD-201). Since the inputs to the stimulus projectors are also represented at the plug board, the lamp driver outputs may be used to operate any combination of 24 stimuli among the four primate chambers.

Thirty-four logic lines from the interface plug board have also been provided to the pigeon and rat laboratories. When the data from these laboratories are too dense or too rapid for collection on paper tape, these data are collected in real time by the computer. This is done by connecting the output signal of any BRS digital logic unit to the plug board lines and routing these signals through the plug board to any of the 32 register inputs. The system interface is presented graphically in Fig. 1.

\section{SOFTWARE}

In addition to the assembler, compilers are provided for FORTRAN IV, ALGOL, and a conversational language called BASIC. Although the ALGOL compiler can function in an $8 \mathrm{~K}$ environment, Hewlett-Packard does not recommend its use without additional memory. The requirement that the BASIC compiler remain in memory generally results in a shortage of memory for our purposes (Ray \& Hogge, 1972). Consequently, the most useful higher-level language has been FORTRAN IV. All drivers, that is, subroutines which interact with $\mathrm{I} / \mathrm{O}$ devices, must be written in assembler. Since most of our programming consists of interactions 
with $\mathrm{I} / \mathrm{O}$ devices and since the initial manipulations of the data are at the level of bits. most of our programming is done using the assembler.

Psychology students can become competent operators of the computer after a few hours of informal instruction. Formal instruction has been directed toward the development of on-line programmers. This instruction has emphasized the assembler both because of the necessity to use the assembler for drivers and because learning to program in assembler results in a closer working relationship with the computer. We have found that the learning of assembler by psychology students need not be as difficult as implied by some (e.g., Durell, 1972). Indeed, students have been able to program the computer on-line within 2 to 3 weeks.

\section{REFERENCES}

Barensten, R. I., \& Lockard, J. S. Minicomputer controller for a behavioral primate laboratory. Behavior Research Methods \&
Instrumentation, 1973, 5, 111-113.

Davenport, J. W., Benson, R. W., Hagguist, W. W., Rankin, G. R. \& Shelton, S. E. Computerized animal intelligence testing. Behavior Research Methods \& Instrumentation, 1972, 4, $67-70$.

Durell, A. B. Fainless computer-controlled experimentation Behavior Research Methods \& Instrumentation, 1972, 4, 165-166.

Moise, S. L. \& Jarrard, L. E. A computer-controlled sy stem for training and testirig primates. Behavior Research Methods \& Instrumentation, 1969, 1, 234-236.

Ray, W. J., \& Hogge, J. H. BASIC subroutines for on-line experiments using Hewlett-Packard computers. Behavior Research Methods \& Instrumentation, 1972, 4, 218.

Sidowski, J. B. Minicomputers, Behavior Research Methods \& Instrumentation, 1970, 2, 267-288.

Sidowski, J. B. Various uses of minicomputers in psychology. Behavior Research Methods \& Instrumentation, 1972, 4 43-50.

Uttal, W. R. Misuse, abuse, overuse, and unuse of on-line computer facilities by psychologists. Behavior Research Methods \& Instrumentation, 1972, 4, 55-60.

(Received for publication October 5, 1973; revision received November $19,1973$. 